\title{
Not a clear cut
}

\author{
Facing budget cuts, the UK's research councils are forced to make unpopular choices. Effective \\ consultations should guide decisions.
}

The Engineering and Physical Sciences Research Council (EPSRC), the UK's main public funding agency for scientific research, found itself in the middle of an intense dispute last month after announcing funding cuts for research in synthetic organic chemistry. The decision, part of a redistribution of financial resources, prompted Anthony Barrett, a chemist at Imperial College London, and more than 100 senior British and international chemists, including several Nobel laureates, to write an open letter opposing the cuts to the country's Prime Minister, David Cameron ${ }^{1}$.

According to a recent study jointly commissioned by the EPSRC and the Royal Society of Chemistry (RSC), the chief representative body of chemists in the UK, about one-fifth of the country's gross domestic product originates from chemistry, with about 6 million jobs supported in $2007^{2,3}$. Cutting its organic chemistry core means therefore undermining an intellectually vital and economically prosperous field, the chemists say in their letter. This simple observation alone raises the question of how these cuts have been decided.

The EPSRC is undergoing a restructuring exercise of its funding portfolio, as it is facing overall budget cuts of about $15 \%$ in real terms in the 2011-2015 period. Currently, the subfield of synthetic organic chemistry takes up $5.6 \%$ (or $£ 44.4$ million) of the total physical sciences and will be reduced by an undetermined amount ${ }^{4}$. The decision to cut the funding for synthetic organic chemistry comes amid the first announcement of this restructuring plan. In a statement replying to the synthetic chemists' concerns, the EPSRC highlights the need to make difficult decisions in a situation of general financial constraints, and in particular the fact that synthetic organic chemistry had enjoyed a period of funding increase in the past few years that has now become unsustainable.

The key point, however, remains.

Although it is possible to be sympathetic with the council's administration in view of its financial restrictions and the resulting need for unpopular cuts, it is the decisionmaking protocol the council has adopted

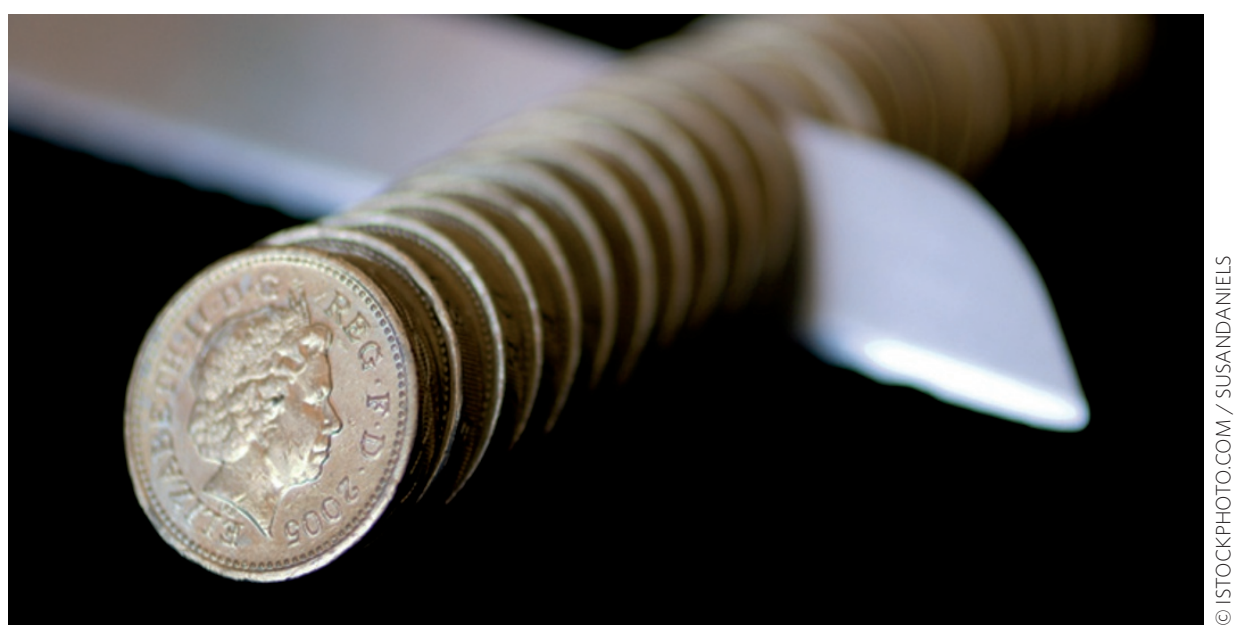

Funding cuts to synthetic organic chemistry in the UK put the EPSRC under fire.

that seems to be nebulous at best. The EPSRC, although relying on advice from a strategic advisory team that includes a dozen professionals from university and industry, does not carry out rounds of formal consultations with learned societies, such as the RSC. This state of affairs, only briefly mentioned in the chemists' letter, must be regarded as the crucial issue, as it will inevitably lead to constant friction between the council and the practitioners.

In this respect, a later letter of concern from the RSC's president, David Phillips, to the EPSRC is correctly trying to shift the debate from the specific funding cuts to the decision-making mechanisms within the council ${ }^{5}$. In particular, the RSC denounces a downright lack of real consultation, having been essentially merely notified about the cuts by the EPSRC.

This is but the last of contentious changes implemented by the EPSRC in the attempt to maximize return for public money spent in science that have angered the academic community of late ${ }^{6}$. The general feeling is that of a perceived heavy top-down control and of a wilful concentration of the bulk of the funding in a few large projects ${ }^{7}$. Decisions on which areas are of strategic importance for the country's economy should actively involve the representative professional bodies in an open and transparent consultation, even at the cost of lengthier discussions among the parties. Prioritization should not disregard areas of lesser immediate economic return, and though not perfect by any means, peer review remains the best way to gauge exploratory scientific research. The most scientifically sound research projects should be funded in all areas. Such flat, bottom-up vision, along the policy lines implemented by the European Research Council for instance ${ }^{8}$, should be in place whenever possible, and scientists have the right to make their voice heard. It is not about lobbying for more money for scientific research, or for synthetic organic chemistry in particular. Or, at least, that is a separate issue. At stake is the overall mechanism of redistribution of taxpayers' money. The UK's scientific community should come together around this point.

At present, the criteria adopted by the EPSRC are far from clear. A more transparent allocation of public resources in scientific research would go a long way towards the common goal of maximizing value for money.
References
1. http://go.nature.com/DlpiC3
2. http://go.nature.com/ILRwal
3. http://go.nature.com/PuyocJ
4. http://go.nature.com/p5qls9
5. http://go.nature.com/Xp8rGX
6. Nature Mater. 8, 535 (2009).
7. Nature Mater. 9, 603 (2010).
8. Nature Mater. 10, 478-479 (2011) 